\title{
Acquired differential regulation of caspase-8 in cisplatin-resistant non-small-cell lung cancer
}

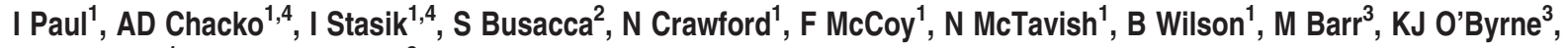 \\ DB Longley ${ }^{1}$ and DA Fennell ${ }^{\star 2}$
}

Failure to efficiently induce apoptosis contributes to cisplatin resistance in non-small-cell lung cancer (NSCLC). Although BCL-2-associated X protein (BAX) and BCL-2 antagonist killer (BAK) are critical regulators of the mitochondrial apoptosis pathway, their requirement has not been robustly established in relation to cisplatin. Here, we show that cisplatin can efficiently bypass mitochondrial apoptosis block caused by loss of BAX and BAK, via activation of the extrinsic death receptor pathway in some model cell lines. Apoptosis resistance following cisplatin can only be observed when both extrinsic and intrinsic pathways are blocked, consistent with redundancy between mitochondrial and death receptor pathways in cisplatin-induced apoptosis. In H460 NSCLC cells, caspase-8 cleavage was shown to be induced by cisplatin and is dependent on death receptor 4, death receptor 5, Fas-associated protein with death domain, acid sphingomyelinase and ceramide synthesis. In contrast, cisplatinresistant cells fail to activate caspase-8 via this pathway despite conserving sensitivity to death ligand-driven activation. Accordingly, caspase-8 activation block acquired during cisplatin resistance, can be bypassed by death receptor agonism. Cell Death and Disease (2012) 3, e449; doi:10.1038/cddis.2012.186; published online 20 December 2012

Subject Category: Cancer

Lung cancer is the leading cause of cancer mortality with over one million new cases diagnosed each year. ${ }^{1}$ The most common type is non-small-cell lung cancer (NSCLC), which accounts for $80 \%$ of all primary lung cancer. ${ }^{2}$ The majority of patients present with advanced disease, whereby surgical resection is of no benefit and systemic therapy forms the mainstay of treatment. ${ }^{3}$ Platinum-based therapy is the mainstay of first line therapy but is associated with high failure with response rates in the region of $20 \%$ observed. ${ }^{4,5}$ In recent years, our understanding of how to treat NSCLC has undergone a paradigm shift initiated by the identification of somatic activating mutations in the epidermal growth factor receptor (EGFR) ${ }^{6,7}$ Lethality relies on the efficient induction of apoptosis involving the BH3-only domain containing protein, BIM. $^{8-11}$ Similarly, ELK4-ALK (anaplastic lymphoma kinase) translocation ${ }^{12}$ is associated with extreme preclinical sensitivity to respective ALK kinase inhibition in both preclinical and clinical settings. Additional molecular subclasses with associated somatic gene alterations have been discovered, predominantly in lung adenocarcinomas and include mutations of BRAF, ${ }^{13}$ human epidermal growth factor receptor $2^{14}$ and phosphoinositide-3-kinase, catalytic, alpha polypeptide. ${ }^{15}$ Despite these advances the majority of patients still fall outside these subclasses and rely on standard cisplatin-based treatment.

Induction of apoptosis is important in mediating tumour response to platinum-based chemotherapy. Defective apoptosis underpins drug resistance and is a hallmark of cancer. ${ }^{16,17}$ Apoptosis can be initiated through two principle core signalling pathways, which are death receptor- or mitochondria-dependent. The mitochondrial apoptotis pathway is regulated by the multidomain pro-apoptotic B-cell lymphoma-2 (BCL-2) family proteins, BCL-2-associated $\mathrm{X}$ protein $(B A X)$ and $B C L-2$ antagonist killer (BAK). These induce permeabilization of the outer mitochondrial membrane upstream of effector caspases. Double knockout of these proteins is sufficient to confer multidrug resistance in mouse embryonic fibroblasts, however, their requirement for the action of cisplatin is less well defined. ${ }^{18,19}$

Altered expression of BAX and BAK has been frequently reported in NSCLC with BAX expression lost in $34-47 \%$ and BAK expression lost in $42-59 \%$ of surgically resected NSCLC tumours. ${ }^{20-25}$ Although these studies failed to demonstrate any prognostic effect of loss of these proteins, analysis was

\footnotetext{
${ }^{1}$ Centre for Cancer Research and Cell Biology, Queen's University Belfast, Northern Ireland, UK; ${ }^{2}$ University of Leicester, Leicester, UK and ${ }^{3}$ Thoracic Oncology Research Group, St James' Hospital, Dublin, Ireland

${ }^{*}$ Corresponding author: DA Fennell, University of Leicester, Hodgkin Building, PO BOX 138, Lancaster Road, Leicester LE1 9HN, UK. Tel: + 44 (0) 1162525174 ; Fax: + 44 (0) 116252 5599; E-mail: df132@le.ac.uk

${ }^{4}$ These authors contributed equally to this work.

Keywords: Apoptosis; BAX; BAK; non-small-cell lung cancer; cisplatin resistance; caspase-8

Abbreviations: ALK, anaplastic lymphoma kinase; ASM, acid sphingomyelinase; BAK, BCL-2 antagonist killer; BAX, BCL-2-associated X protein; BCL-2, B-cell lymphoma-2; BID, BCL-2 interacting domain death agonist; BIM, BCL2-interacting mediator of cell death-associated recruitment domain; CFLIP, cellular FLICE inhibitory protein; DR4, death receptor 4; DR5, death receptor 5; EML4, echinoderm microtubule-associated protein-like 4; FADD, Fas-associated protein with death domain; FLICE, FADD-like interleukin-1 $\beta$-converting enzyme; HER2, human epidermal growth factor receptor 2; PARP, poly (ADP-ribose) polymerase; PIKC3A, phosphoinositide-3-kinase, catalytic, alpha polypeptide; $\mathrm{R}_{8} B I D$, polyarginine tagged BID; RNA, ribose nucleic acid; shRNA, short hairpin RNA; TRAIL, TNF receptorrelated apoptosis inducing ligand

Received 16.6.11; revised 21.9.12; accepted 21.9.12; Edited by A Stephanou
} 
carried out for each gene in isolation and the majority of patients did not receive adjuvant therapy. Given that BAX and BAK are frequently lost in NSCLC, we hypothesised that loss of both BAX and BAK in NSCLC could potentially mediate profound chemoresistance. In contrast, however, our findings reveal that $\mathrm{BAX}$ and $\mathrm{BAK}$ are dispensable for cisplatinmediated apoptosis in some model systems, where generation of ceramide can induce apoptosis via caspase-8. Moreover, in cisplatin-resistant NSCLC cells, although both the intrinsic apoptotic pathway and caspase-8 activation are impaired in response to cisplatin, sensitivity to death receptor activation is still conserved.

\section{Results}

Silencing BAX and BAK in $\mathrm{H} 460$ and $\mathrm{H} 1299$ cells results in mitochondrial apoptosis block. In order to determine if mitochondrial apoptosis block would confer resistance to cisplatin-induced apoptosis in NSCLC cells, we generated H460 and H1299 clones expressing short hairpin RNAs
(shRNAs) simultaneously targeting BAX and BAK. Pairs of clones with different $B A X$ and $B A K$ targeting sequences were generated (sh-BAXBAK) and comparisons made with stably expressing non-targeting (NT) short hairpin RNA (shRNA) clones (sh-NTNT). BAX and BAK protein expression in $\mathrm{H} 460$ clones sh-BAXBAK $1 \mathrm{C}$ and $4 \mathrm{~F}$ was undetectable and in $\mathrm{H} 1299$ clones sh-BAXBAK $1 \mathrm{~F} 1 \mathrm{~A}$ and $2 \mathrm{~A} 4 \mathrm{~B}$ was considerably reduced as assessed by western blotting (Figure 1a).

We next confirmed that the level of BAX and BAK silencing in our clones was sufficient to block mitochondrial apoptosis. All sh-BAXBAK clones showed inhibition of caspase-9 cleavage to the p37 form following $24 \mathrm{~h}$ treatment with staurosporine, versus sh-NTNT controls (Figure 1b). Caspase-3 activation was also significantly attenuated after staurosporine in the sh-BAXBAK clones of both $\mathrm{H} 460$ and H1299 cells compared with the respective sh-NTNT controls (Figure 1c). The H460 sh-BAXBAK clones also exhibited resistance to pro-apoptotic peptides corresponding to the $\mathrm{BH} 3$ domain of the pro-apoptotic protein BID (BH3 ${ }^{\mathrm{BID}}$ ) both at isolated mitochondria and whole-cell level, as we recently a

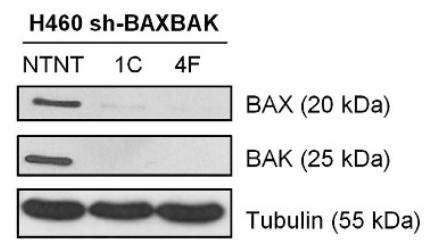

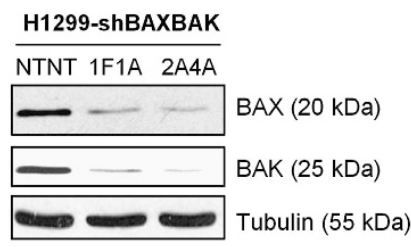

b

H460 sh-BAXBAK

Staurosporine

$(\mathrm{nM})$

\begin{tabular}{|c|c|c|c|c|c|c|c|c|}
\hline \multicolumn{9}{|c|}{ H460 sh-BAXBAK } \\
\hline \multicolumn{3}{|c|}{ NTNT } & \multicolumn{3}{|c|}{ BAXBAK $1 \mathrm{C}$} & \multicolumn{3}{|c|}{ BAXBAK 4F } \\
\hline C & 100 & 250 & C & 100 & 250 & C & 100 & 250 \\
\hline
\end{tabular}
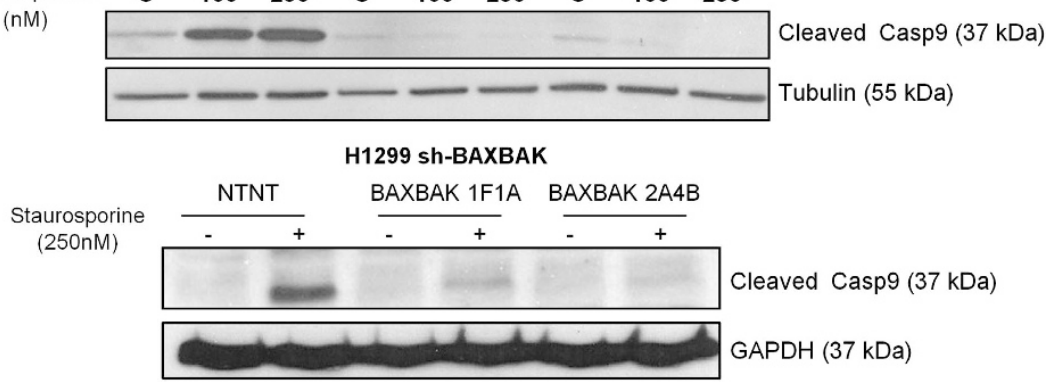

C

H460 sh-BAXBAK

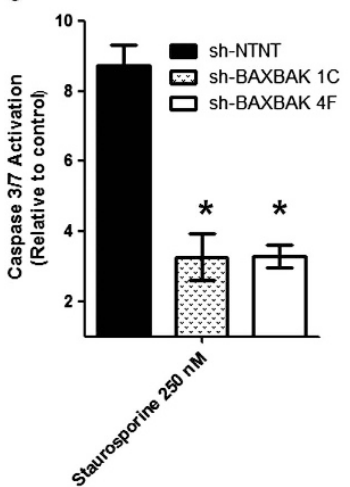

H1299 sh-BAXBAK

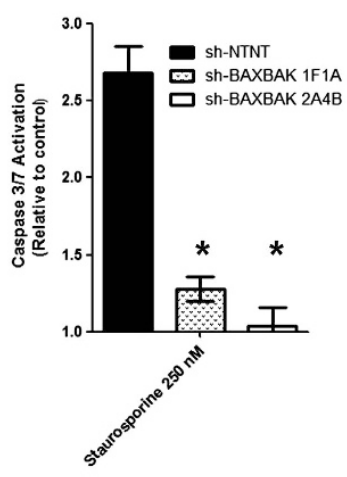

Figure 1 BAXBAK silenced NSCLC cells have mitochondrial apoptosis block. (a) Western blot showing BAX and BAK protein expression in H460 and H1299 sh-BAXBAK clones compared with corresponding sh-NTNT controls. (b) Western blot for caspase-9 following $24 \mathrm{~h}$ treatment with indicated doses of staurosporine in sh-NTNT and shBAXBAK clones from H460 and H1299 cells. (c) Luminescent DEVD-ase assay measuring caspase-3-like activity in sh-NTNT and sh-BAXBAK cells from H460 and H1299 shown relative to untreated control. Data are expressed as mean \pm S.D. $\left({ }^{*} P<0.001\right)$ 
reported. ${ }^{26}$ Together these data show that stable knockdown of BAX and BAK using short hairpin RNA interference in $\mathrm{H} 460$ and $\mathrm{H} 1299$ cells results in effective suppression of mitochondrial apoptotic signalling.

Cisplatin efficiently induces apoptosis in $\mathrm{H} 460$ shBAXBAK cells but not H1299 sh-BAXBAK cells. Having demonstrated mitochondrial apoptosis block in both cell lines in response to staurosporine (Figures $1 \mathrm{~b}$ and $\mathrm{c}$ ) and $\mathrm{R}_{8} \mathrm{BID},{ }^{26}$ we wanted to study the effect that this block would have on cisplatin-induced apoptosis. H460 sh-BAXBAK cells showed no attenuation in apoptosis after treatment with cisplatin, as evidenced by sub- $G_{0} / G_{1}$ fraction (Figure 2a). In contrast, H1299 sh-BAXBAK clones showed statistically significant attenuation of apoptosis induction, as evidenced by sub- $\mathrm{G}_{0} / \mathrm{G}_{1}$ fraction, compared with sh-NTNT control cells (Figure $2 \mathrm{~b}$ ). These results were confirmed by immunoblot detection of poly (ADP-ribose) polymerase (PARP) cleavage (Supplementary Figure S1). To understand what could be the reason for this difference in apoptotic response, we assessed activation of caspase-3. H460 sh-BAXBAK cells showed no attenuation in caspase-3 activity after cisplatin treatment, whereas a significant reduction was observed in H1299 sh-BAXBAK clones (Figure 2c). Together, these data show that cisplatin can induce apoptosis independent of BAX and BAK in H460 cells, however, in H1299 cells, cisplatin-induced apoptosis is $B A X / B A K-d e p e n d e n t$.

Cisplatin induces caspase-8 cleavage in a panel of NSCLC cell lines and H460 sh-BAXBAK clones, but not in $\mathrm{H} 1299$ cells. It has been shown previously that chemotherapy treatment has effects on the death receptor pathway by sensitising a number of different cell types to TNF receptor-related apoptosis inducing ligand (TRAIL). ${ }^{27,28}$ It has also been reported that cisplatin leads to Fas receptor clustering and activation independent of ligand binding. ${ }^{29}$ This led us to hypothesise that cisplatin induces apoptosis independently of BAX and BAK through its effects on caspase-8, the initiator caspase of the death receptor pathway. Accordingly, caspase-8 cleavage in response to cisplatin was assessed in a panel of NSCLC lines. Cleavage of procaspase-8 to p41/43-caspase-8 was detected by western blotting in all four cell lines studied, in a dose-dependent manner, after $24 \mathrm{~h}$ exposure to cisplatin (Figure 3a). To validate these data, caspase-8 activity assays were performed, showing caspase- 8 activity in these cells in response to cisplatin (Supplementary Figure S8). Together these data suggest that cisplatininduced caspase-8 activity is a common feature in NSCLC cell lines.

Next, we assessed caspase-8 cleavage following cisplatin treatment in the sh-BAXBAK and sh-NTNT clones as a possible explanation for the difference observed in caspase-3 activity in sh-BAXBAK cells. In H460 cells, caspase- 8 was cleaved in both sh-NTNT and sh-BAXBAK clones. Caspase-8 cleavage, however, was not detected in any of the H1299 clones (Figure 3b). Again these data were supported by similar findings using the caspase-8 activity assay (Supplementary Figure S9). a

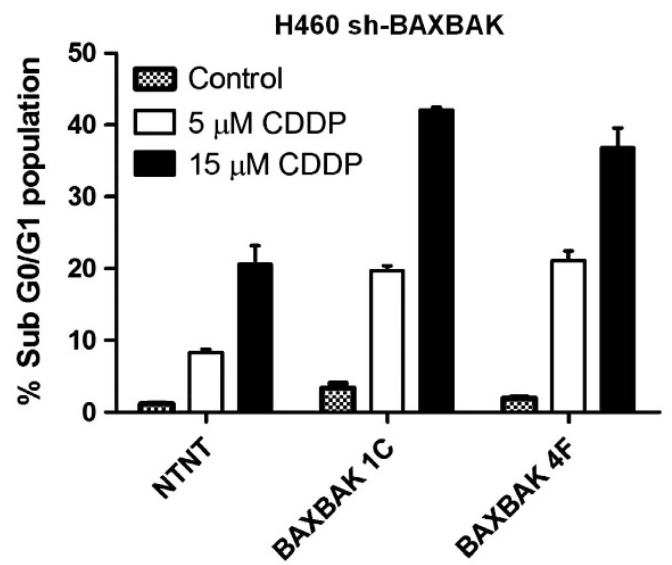

b
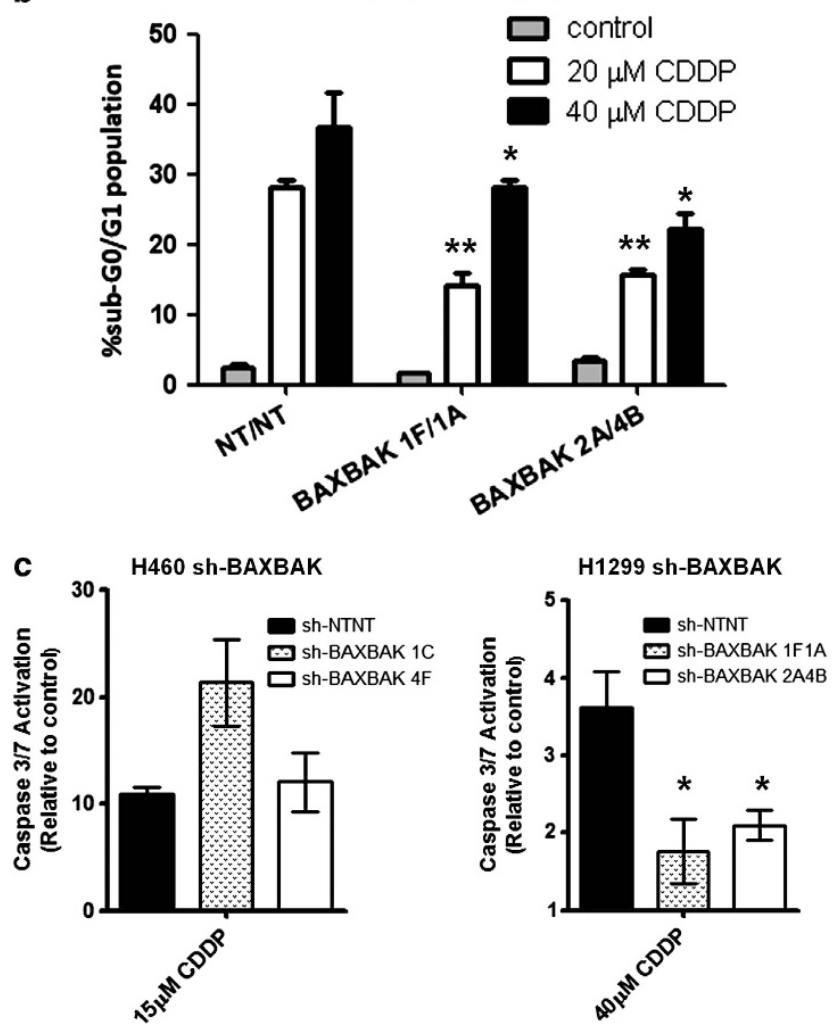

Figure 2 Cisplatin induces apoptosis in $\mathrm{H} 460$ sh-BAXBAK cells but not $\mathrm{H} 1299$ sh-BAXBAK cells. (a) Flow cytometry of PI-stained $\mathrm{H} 460$ clones after $48 \mathrm{~h}$ treatment with cisplatin. Apoptotic cells are indicated by increase in sub-G0/G1 population. (b) Flow cytometry of of Pl-stained $\mathrm{H} 1299$ clones after $48 \mathrm{~h}$ treatment with cisplatin. Apoptotic cells are indicated by increase in sub-G0/G1 population $\left({ }^{*} P<0.001\right.$, ${ }^{* *} P<0.01$ ). Data are expressed as mean \pm S.D. (c) Luminescent DEVD-ase assay measuring caspase-3-like activity in sh-NTNT and sh-BAXBAK cells from H460 and $\mathrm{H} 1299$ after $24 \mathrm{~h}$ treatment with cisplatin, shown relative to untreated controls. Data are expressed as mean \pm S.D. $\left({ }^{*} P<0.001\right)$

Caspase-8 silencing rescues $\mathrm{H} 460$ sh-BAXBAK cells but not sh-NTNT control cells from cisplatin-induced apoptosis. Cisplatin-induced caspase-8 cleavage in H460 cell line was sufficient to drive apoptosis independent of BAX and BAK, contrary to $\mathrm{H} 1299$ cells, where caspase-8 cleavage was not seen after cisplatin treatment, implicating BAXBAK 


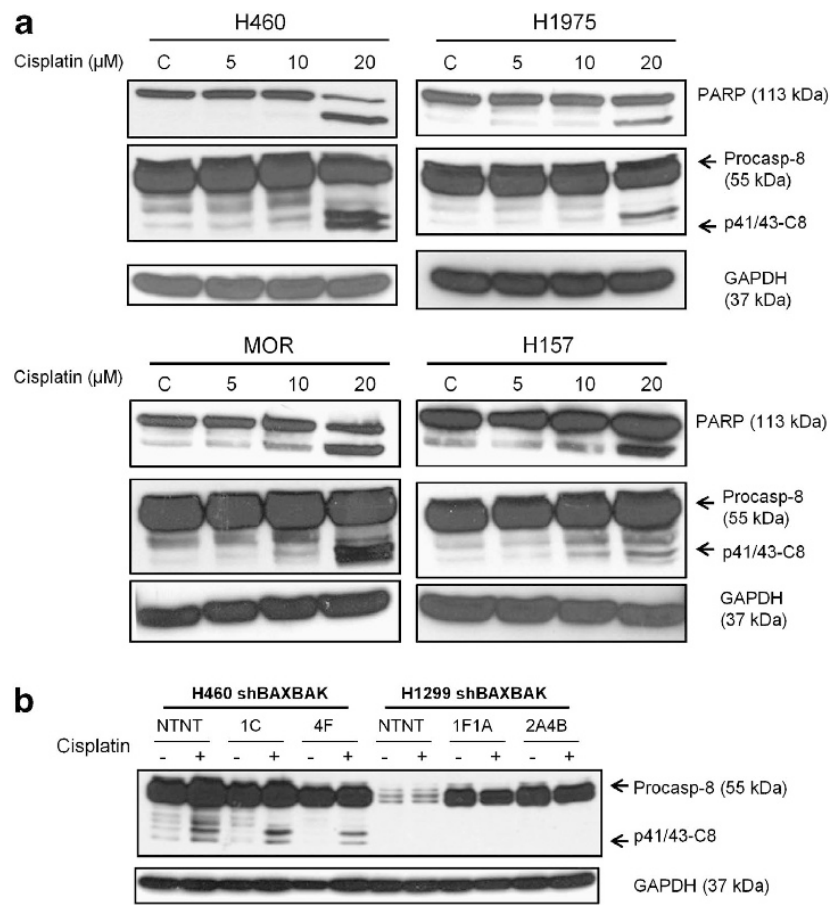

Figure 3 Cisplatin induces caspase- 8 cleavage in a panel of NSCLC cells but not in $\mathrm{H} 1299$ cells. (a) Western blots showing processing of PARP and caspase-8 in a panel of NSCLC cell lines with increasing doses of cisplatin for $24 \mathrm{~h}$. (b) Western blot for caspase-8 in H460 and H1299 sh-BAXBAK cells with matched sh-NTNT controls after $24 \mathrm{~h}$ treatment with cisplatin (H460 $15 \mu \mathrm{M}, \mathrm{H} 129940 \mu \mathrm{M})$

dependence. To confirm whether caspase-8 was required for apoptosis signalling in the absence of BAXBAK, we silenced caspase-8 expression in H460 sh-BAXBAK clones using transient siRNA transfection. TRAIL treatment was included as a positive control to confirm the level of caspase-8 silencing was sufficient to block death ligand/death receptordriven apoptosis. Significant attenuation in cisplatin-induced apoptosis was noted in both $\mathrm{H} 460$ sh-BAXBAK clones studied (1C and 4F) in the caspase-8-silenced cells compared with scrambled control siRNA (Figure 4a). Attenuation in caspase-3 activation was also detected (data not shown). However, in control sh-NTNT cells silencing of caspase-8 did not prevent sh-NTNT cells and apoptosis induction (Figure 4b). These data show that redundancy can exist between intrinsic and extrinsic apoptosis pathways in response to cisplatin. In H1299 cells, the death receptor pathway is dysfunctional as evidenced by their resistance to rhTRAIL. ${ }^{30}$ This was associated with reduced procaspase-8, death receptor 4 (DR4) and death receptor 5 (DR5) expression as compared with $\mathrm{H} 460$ cells (Figure $3 \mathrm{~b}$ and Supplementary Figure S2).

To determine if cisplatin-induced caspase- 8 cleavage was dependent on formation of the death inducing signalling complex (DISC), its components were silenced in $\mathrm{H} 460$ shBAXBAK cells and cisplatin-induced caspase-8 cleavage was assessed (Figure 4c). Silencing of the death receptors, DR4 and DR5 did not affect generation of p41/43-caspase-8, but resulted in partial attenuation of p18-caspase-8 generation after cisplatin. This correlated with attenuation in PARP cleavage. Furthermore, silencing Fas-associated protein with death domain (FADD) resulted in marked attenuation of $p 41 /$ 43-caspase-8 and no detectable p18-caspase-8 after $24 \mathrm{~h}$ cisplatin treatment. Again this correlated with attenuation of PARP cleavage (Figure 4c). Additionally, caspase-3/7 activation was reduced following silencing of either one of the DISC components (Supplementary Figure S3). Similar results were also observed in both H460 sh-BAXBAK $1 \mathrm{C}$ and shBAXBAK4F clones (not shown). In contrast, Fas silencing had no effect on cisplatin-induced apoptosis (Supplementary Figure S4).

Cisplatin-induced procaspase-8 cleavage is acid sphingomyelinase (ASM)-dependent. It has been shown previously that cisplatin can induce ceramide generation. ${ }^{31}$ We therefore investigated the role of ASM in controlling caspase8 activation by cisplatin in $\mathrm{H} 460$ sh-BAXBAK clones. Pretreatment of cells with imipramine, a known inhibitor of ASM, resulted in an attenuation of p18-caspase-8 generation following treatment with cisplatin (Figure 5a). Similar results were seen when ASM expression was silenced using siRNA, as assessed by western blotting (Figure $5 b$ ). To establish whether cisplatin-induced caspase-8 cleavage was ASMdependent, we studied this effect in other NSCLC cell lines. In MOR and $\mathrm{H} 1975$ cells pretreated with imipramine, we observed reduced p41/43-caspase-8 following cisplatin treatment; $p 18$-caspase- 8 was not detectable in these cell lines (Figure 5c). In addition, cisplatin-induced caspase-8 activity was suppressed with imipramine in H460 MOR and H157 cells (Supplementary Figure S10) Ceramide clusters were observed $24 \mathrm{~h}$ after cisplatin treatment in $\mathrm{H} 460$ cells, but these were abolished following pre-treatment with imipramine (Supplementary Figure S5). Together these data confirm that ASM, responsible for ceramide generation following cisplatin treatment, induces caspase- 8 activation in both parental and shBAX/BAK cell lines. Accordingly, imipramine strongly attenuated caspase-3 activation following cisplatin treatment in shBAX/BAK cells (Supplementary Figure S6).

In cisplatin-resistant cells, caspase- 8 can be activated by TRAIL, but not by cisplatin. We have previously shown that cisplatin-resistant cells harbour defective mitochondrial signalling in response to cisplatin, owing to suppression of $\mathrm{BH} 3$ death signals, whereas there is conservation of $\mathrm{BAX} /$ BAK sensitivity to exogenous $\mathrm{BH} 3$ domains. ${ }^{32}$ We therefore decided to investigate whether an analogous dysregulation of the death receptor pathway signalling occurs in cisplatinresistant NSCLC. In both H460 cisplatin-resistant (CISPR) cells developed in our laboratory (as described previously by Crawford et $a .^{32}$ ) and MOR CISPR cells, ${ }^{33}$ no caspase-8 cleavage was observed following cisplatin treatment, although caspase-8 cleavage and apoptosis could be efficiently induced by TRAIL (Figure 6a). Thus, the death receptor apoptosis machinery is present in H460 CISPR and MOR CISPR cells and remains functional in response to receptor activation. Indeed, ceramide expression was shown to increase in response to cisplatin in the $\mathrm{H} 460$ parental but not the $\mathrm{H} 460$ cisplatin-resistant cells (Figure 6b). This was 
a
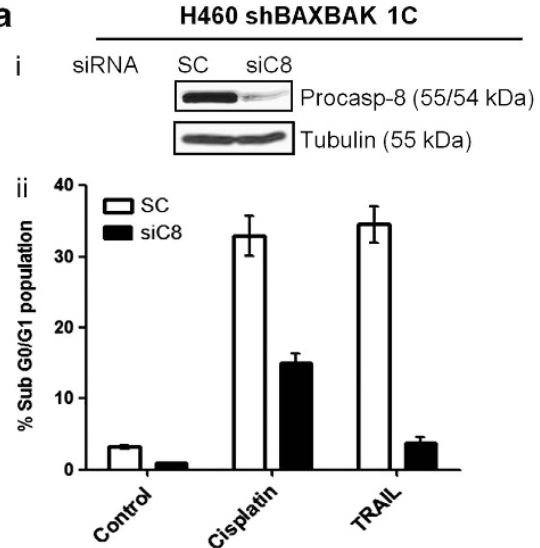

H460 shBAXBAK 4F
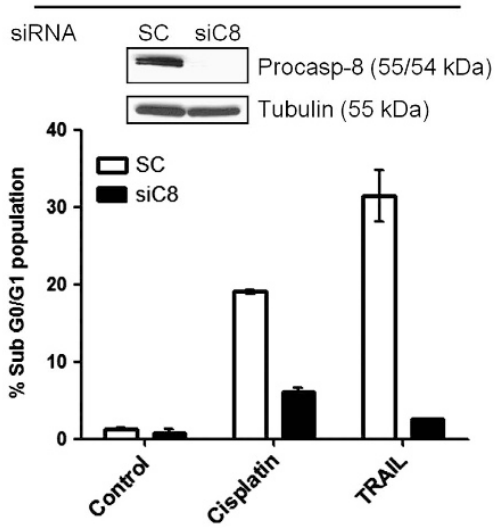

b

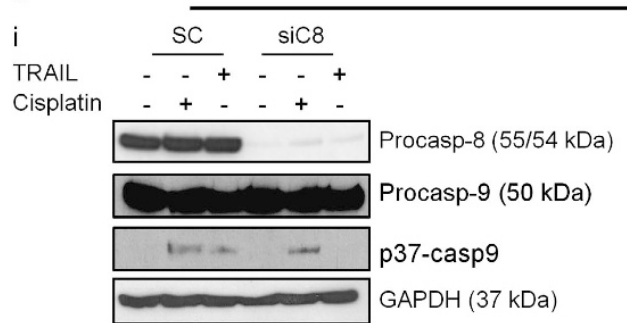

H460 shNTNT

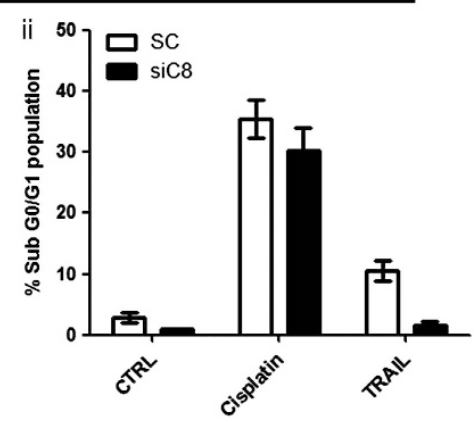

$\begin{array}{lllllll}\text { siRNA } & \text { SC } & \text { DR4 } & \text { DR5 } & \text { FADD } \\ \text { Cisplatin } & - & + & + & + & - & +\end{array}$

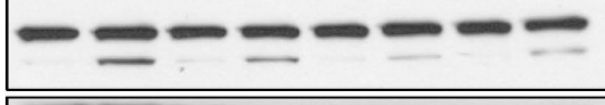

PARP (113 kDa)

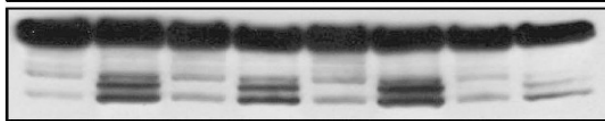

Procasp-8

(55 kDa)

$\leftarrow$ p41/43-C8

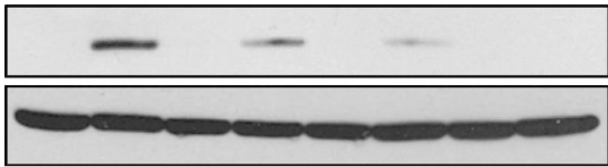

p18-C8

a-Tubulin

(55kDa)
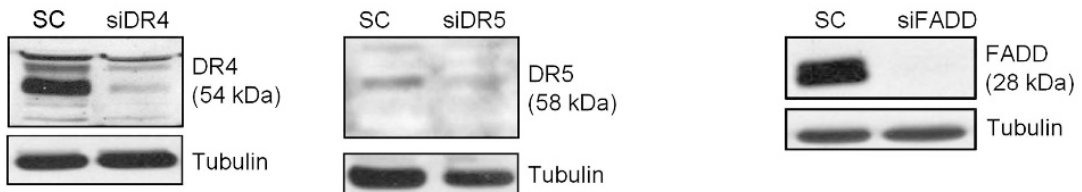

Figure 4 Caspase-8 mediates cisplatin-induced apoptosis in H460 sh-BAXBAK cells but not sh-NTNT control cells. (a) (i) Western blot showing siRNA-mediated silencing of caspase-8 in H460 sh-BAXBAK clones. (ii) Flow cytometry of PI-stained H460 sh-BAXBAK clones, transfected for $48 \mathrm{~h}$ with caspase-8 siRNA (siC8) or scrambled control $(\mathrm{SC})$ and treated with cisplatin $(5 \mu \mathrm{M})$ or TRAlL $(10 \mathrm{ng} / \mathrm{ml})$ for $48 \mathrm{~h}$. Apoptotic cells are indicated by increase in sub-G0/G1 population. Data are expressed as mean \pm S.D. (b) (i) Western blot for caspase-8 and caspase-9 in H460 sh-NTNT cells after transfection for $48 \mathrm{~h}$ with C8 siRNA and treatment with cisplatin or TRAIL. Caspase-8 silencing attenuates TRAIL induced but not cisplatin-induced caspase-9 cleavage. (ii) Flow cytometry of PI-stained H460 sh-NTNT cells, transfected for $48 \mathrm{~h}$ with caspase-8 siRNA (siC8) or scrambled control (SC) and treated with cisplatin $(15 \mu \mathrm{M})$ or TRAIL $(10 \mathrm{ng} / \mathrm{ml})$ for $48 \mathrm{~h}$. Data are expressed as mean \pm S.D. (c) Western blots for PARP, caspase-8, DR4, DR5, FADD. Caspase-8 and PARP cleavage, induced by cisplatin, is attenuated after siRNA-mediated silencing of DR4, DR5 and FADD in H460 sh-BAXBAK $1 C$

also observed in the MOR CISPR cells (not shown). However, this block in ceramide generation was not due to a block in ASM enzyme activity per se, as we detected no difference in either basal, or cisplatin-induced ASM activity between resistant and parental cells (Figure 6c). This suggests that defective ASM is not responsible for failure of caspase- 8 cleavage in response to cisplatin in resistant cells. 

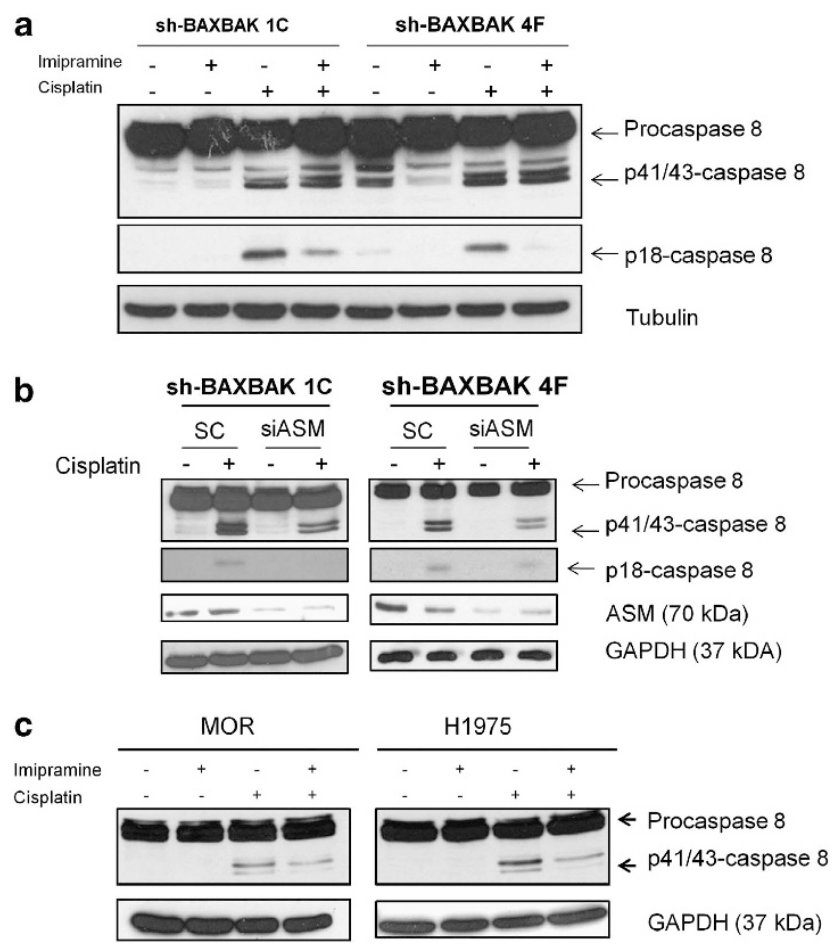

Figure 5 Cisplatin-induced caspase-8 cleavage is acid sphingomyelinase (ASM)-dependent. (a) Western blot for caspase-8 after $2 \mathrm{~h}$ treatment with imipramine $(50 \mu \mathrm{M})$ and $24 \mathrm{~h}$ cotreatment with cisplatin $(5 \mu \mathrm{M})$ in $\mathrm{H} 460$ shBAXBAK cells. (b) Western blot for caspase-8 and ASM. (c) Western blot for caspase-8 from MOR and $\mathrm{H} 1975$ treated as in (a) showing imipramine attenuates cisplatin-induced caspase-8 cleavage in these NSCLC cell lines

\section{Discussion}

BAX and BAK are key regulators of the mitochondrial apoptosis pathway as evidenced by knockout of BAX and BAK in mouse embryonic fibroblasts. ${ }^{19}$ Their frequency of loss in NSCLC prompted us to investigate whether functional mitochondrial signalling was required for activity of cisplatin, the most commonly used drug in the treatment of NSCLC. To achieve this, we studied the effect of cisplatin in BAX/BAKsilenced NSCLC cell lines and observed two different phenotypes. In H460, caspase-8 cleavage was observed in response to cisplatin, sufficient to induce apoptosis independent of BAX and BAK. In contrast, H1299 cells showed no evidence of caspase- 8 cleavage in response to cisplatin and so were unable to bypass mitochondrial apoptosis block induced by loss of BAX and BAK. This is in agreement with other reports showing that $\mathrm{H} 1299$ cells are resistant to ligand stimulation of the death receptor pathway. ${ }^{30}$ Blocking death receptor-mediated apoptosis, through depletion of caspase-8 by RNA interference, was sufficient to rescue H460 shBAXBAK, but not sh-NTNT cells from cisplatin, demonstrating redundancy between the two apoptotic pathways following cisplatin treatment.

This is the first report to show that cisplatin can induce apoptosis through a BAX/BAK-independent, ASM/caspase-8/ FADD-dependent fashion. This is contrary to our initial hypothesis based on previous reports in BAX/BAK knockout
MEFs. Moreover, we found that sh-BAXBAK H460 cells were more sensitive to cisplatin than parental cells. This could be because of an impairment of mitochondrial network dynamics and metabolism occurring after BAX/BAK depletion (for review see Auret and Martin ${ }^{34}$ ), that could in turn lead to increased sensitivity to toxic effect of the drug despite block of caspase-9 activation following staurosporine. Although bypass of the mitochondrial pathway was only observed in the H460 sh-BAXBAK model, treatment of a panel of NSCLC cell lines showed caspase-8 activation following cisplatin in all, except $\mathrm{H} 1299$, suggesting that this is a potentially common process in NSCLC. This is not surprising given our previous report that procaspase- 8 is overexpressed in $85 \%$ of NSCLC compared with matched normal lung tissue. ${ }^{35}$

The finding that BAX and BAK are not essential for effective apoptosis induction, led us to investigate the mechanism regulating the mitochondrial bypass pathway and its potential alteration in resistance to cisplatin. In both cell lines studied, H460 and MOR, cisplatin-induced caspase- 8 cleavage and ceramide accumulation were abrogated in cisplatin-resistant (CISPR) cells, but not in parental cells. However, the death receptor pathway apparatus remained functional in both cisplatin-resistant daughter cell lines, as evidenced by caspase-8 cleavage, PARP cleavage and G0/G1 fraction after treatment with rhTRAIL. This would suggest absence of an upstream death signal initiating apoptosis via the death receptor pathway. The importance of ceramide generation via ASM for both cisplatin-induced apoptosis and death receptordependent apoptosis has been demonstrated previously. ${ }^{36-38}$ Our data confirm generation of ceramide in response to cisplatin in parental cells, but not in cisplatin-resistant cells and this is not due to dysfunctional ASM in the resistant cells. Although ASM has been shown to regulate cisplatin-induced ceramide generation, production of ceramide requires auxiliary factors. It is possible that an as yet undefined functional defect in one or more of these factors may explain lack of cisplatin-induced ceramide generation in cisplatin-resistant NSCLC cells. From a translational perspective, our data suggest that in cisplatin-resistant cells, despite the absence of ceramide generation and subsequent caspase-8 signalling in response to cisplatin, DISC activation can still be achieved by ligand stimulation of the death receptors with rhTRAIL.

In conclusion, cisplatin resistance requires a block in both mitochondrial and a parallel, independent pathway involving ASM/FADD/caspase-8. Importantly, selection for cisplatin resistance leads to a block in death receptor apoptosis. These cells, however, do not exhibit cross-resistance to death receptor-targeted therapies such as TRAIL and silencing of cFLIP (data not shown). These findings therefore support a rationale for targeting the death receptor pathway, as a strategy for bypassing platinum resistance.

\section{Materials and Methods}

Reagents, cytotoxics. Recombinant human TRAIL was purchased from Calbiochem (Gibbstown, NJ, USA), Imipramine and staurosporine were purchased from Sigma-Aldrich (St Louis, MO, USA); cisplatin was obtained from Mayne Pharma, Adelaide, SA, Australia.

Cell culture. ATCC NCl-H1975, NCl-H460, NCl-H1299 and MOR non-smallcell lung cancer (NSCLC) cells were grown in Roswell Park Memorial Institute (RPMI) 1640 medium supplemented with $10 \%$ foetal bovine serum and $50 \mu \mathrm{g} / \mathrm{ml}$ 

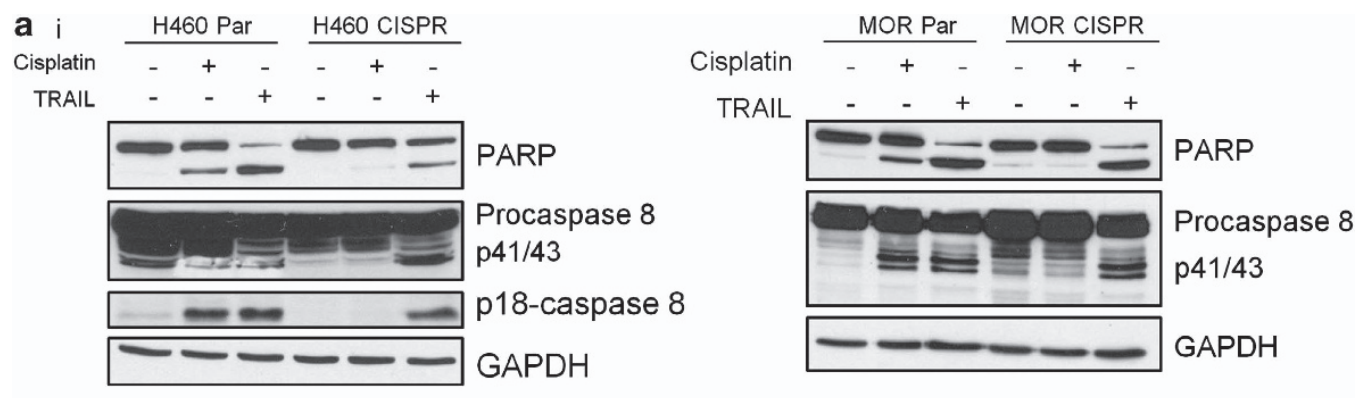

ii
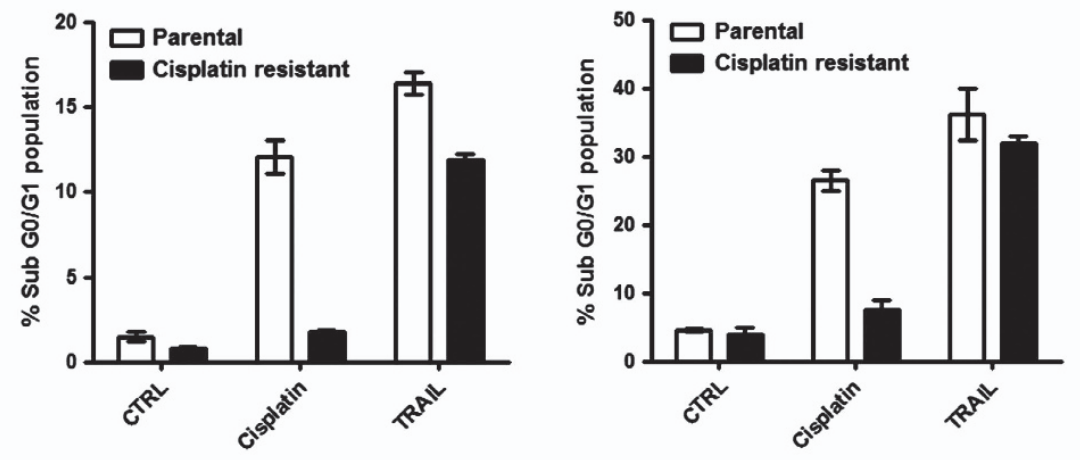

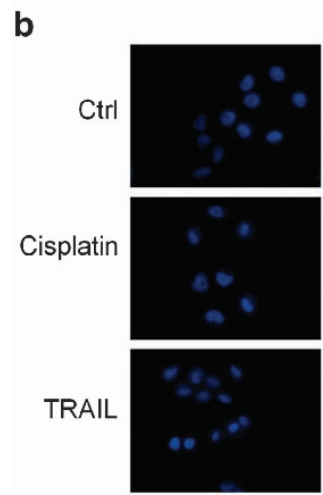

DAPI
H460 Parental
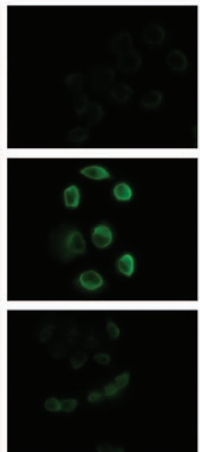

Ceramide
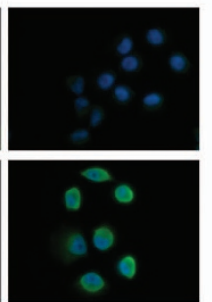

H460

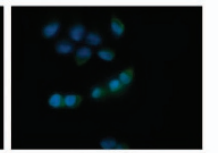

Merge

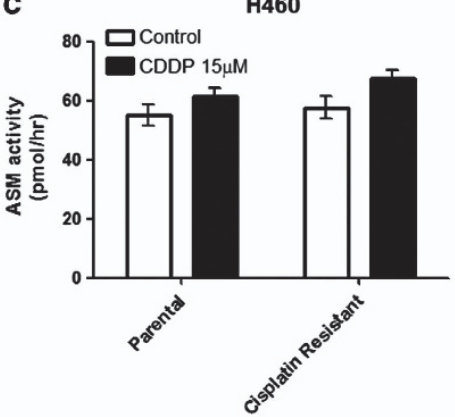

H460 CISPR

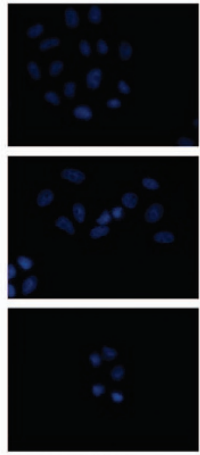

DAPI

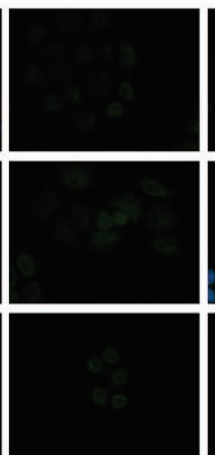

Ceramide

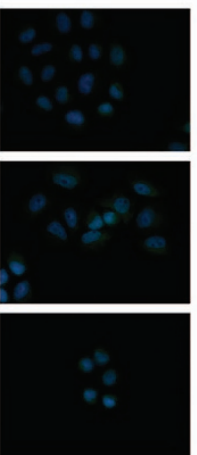

Merge
MOR

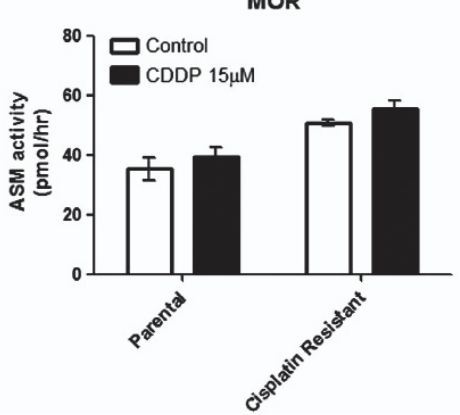

Figure 6 Caspase-8 activation is blocked in cisplatin-resistant cells but can be activated by TRAIL. (a) (i) Western blot for PARP and caspase-8 in H460 and MOR paired parental (Par) and cisplatin-resistant (CISPR) cells after $24 \mathrm{~h}$ cisplatin $(15 \mu \mathrm{M})$ or TRAIL (10 ng/ml) treatment. (ii) Flow cytometry of PI-stained H460 and MOR paired parental (Par) and cisplatin-resistant (CISPR) cells after $24 \mathrm{~h}$ cisplatin $(15 \mu \mathrm{M})$ or TRAIL $(10 \mathrm{ng} / \mathrm{ml})$ treatment. Data are expressed as mean \pm S.D. (b) Representative images of cells stained for ceramide (green) in H460 Par and CISPR cells. Cells were treated for $24 \mathrm{~h}$ with cisplatin ( $15 \mu \mathrm{M})$ or $4 \mathrm{~h}$ with TRAIL (10 ng/ml). (c) Fluorescent ASM activity assay shows PAR and CISPR cells for H460 and MOR have equivalent baseline levels of ASM activity with a small increase after $24 \mathrm{~h}$ cisplatin treatment

penicillin/streptomycin (Invitrogen, Paisley, UK). NCl-H157 NSCLC cells were maintained in Dulbecco's modified Eagle's medium supplemented with $10 \%$ foetal bovine serum, $1 \mathrm{mM}$ sodium pyruvate and $50 \mu \mathrm{g} / \mathrm{ml}$ penicillin/streptomycin. All cell lines were maintained in a $5 \% \mathrm{CO}_{2}$ incubator at $37^{\circ} \mathrm{C}$.
Generation of BAX and BAK-silenced NSCLC cell lines. Plasmids encoding shRNA sequences to BAX, BAK and NT shRNA (SA Biosciences, Frederick, MD, USA) were transfected into $\mathrm{H} 460$ and H1299 NSCLC cells using FuGene 6 reagent (Roche, Burgess Hill, West Sussex, UK) according to 
manufacturer's protocol. BAX plasmids with G418 resistance selection marker, BAK plasmids with puromycin resistance selection marker and separate NT plasmids bearing either G418 or puromycin resistance selection markers were used. Cells were transfected cells in six-well plates and were incubated with either G418 (H460-0.4 mg/ml, H1299-0.8 mg/ml) (Sigma-Aldrich) or $2 \mu \mathrm{g} / \mathrm{ml}$ puromycin (Calbiochem). Discrete colonies were selected and screened by western blotting. Cells stably expressing shRNA targeting BAX were then re-transfected with shRNA targeting BAK to generate clones with stable knockdown of both BAX and BAK.

siRNA transfections. The non-silencing control (SC) siRNA, caspase-8targeted (C8), FADD-targeted, DR5-targeted and DR4-targeted siRNA sequences were the same as previously described. ${ }^{35,39}$ siRNAs for in vitro transfection were obtained from Dharmacon (Chicago, IL, USA). SMARTpool ON-TARGET plus ASM (SMPD1) siRNA was obtained from Dharmacon. Reverse siRNA transfections were carried out using LipofectamineRNAi max reagent (Invitrogen) according to the manufacturer's instructions.

Western blotting. Twenty-five micrograms of cell lysate were loaded and resolved by SDS-PAGE as previously described. ${ }^{40}$ Primary antibodies used were: anti-caspase-8 (Alexis Biochemicals, San Diego, CA, USA), anti-PARP (eBioscience, San Diego, CA, USA), anti-GAPDH (Serotec, Kidlington, UK), anti-ASM, anti- $\alpha$-tubulin (Abcam, Cambridge, MA, USA), anti-BAX, anti-BAK, anti cleaved caspase-9 (Cell Signalling Technology Inc., Danvers, MA, USA) anti-DR4 (Calbiochem), anti-DR5 (Santa Cruz Biotechnology, Santa Cruz, CA, USA) and FADD (BD Bioscience Oxford, Oxfordshire, UK). Secondary antibodies used were: goat anti-mouse HRP (DAKO P0447, DAKO Ely, Cambridgeshire, UK) and goat anti-rabbit HRP (DAKO P0448).

Caspase activity assay (8 and $3 / 7$ ). For caspase activity assay, cells were seeded at $5000 /$ well in a white-walled 96 -well plate $24 \mathrm{~h}$ before start of experiment. Media was then replaced with drug concentrations as shown in the text. Imipramine was added $2 \mathrm{~h}$ before cisplatin, where required. DEVD-ase activity was determined after $24 \mathrm{~h}$ incubation with Caspase-glo luminescent assay (Promega, Southampton, UK) according to manufacturer's instructions, using a Berthold luminometer with $1 \mathrm{~s}$ integration time.

Ceramide immunostaining. Cells were seeded onto a glass coverslip inside a six-well plate at 150000 cells per well, $24 \mathrm{~h}$ before start of experiment. Cisplatin and/or imipramine were applied as described previously, and after $24 \mathrm{~h}$ cells were fixed in $2 \%$ paraformaldehyde. Coverslips were incubated with anticeramide antibody (Alexis) followed by goat anti-mouse AlexaFluor488 secondary antibody (Invitrogen) and mounted on slides with Pro-long gold mountant with DAPI (Invitrogen). Images were captured on a Leica DM microscope (Leica Microsystems, Wetzlar, Germany) using a $\times 40$ objective lens.

Flow cytometry. Cells were seeded $48 \mathrm{~h}$ post transfection with siRNA as described, at a density of $1 \times 10^{5}$ cells per well in six-well plates. Cells were treated after $24 \mathrm{~h}$ and allowed to grow for a further $48 \mathrm{~h}$. Following treatment with cisplatin, DNA content was evaluated by propidium iodide staining of cells using a FACS Calibur Flow Cytometer (Becton Dickinson, Franklin Lakes, NJ, USA), in the FL-2 channel to determine percentage of cells with DNA content $<2 \mathrm{~N}$.

Surface expression of death receptors was assessed following live cell staining with one of the phycoerythrin-conjugated monoclonal antibodies: DR4, DR5, Fas, DcR2 (all from eBioscience) or TNFR1 antibody (R\&D Systems, Minneapolis, MN, USA). In all experiments, isotype control (mouse $\lg _{1}$ from eBioscience) was used.

ASM activity assay. ASM activity was determined using an ASM activity assay kit (Echelon Biosciences Inc., Salt Lake City, UT, USA) according to the manufacturer's protocol. Cells were disrupted using a microprobe sonicator (Sonics Vibra-Cell VC 130 Ultrasonic Homogeniser) with 10 pulses ( $2 \mathrm{~s}$ on, $1 \mathrm{~s}$ off) at $30 \%$ output. $30 \mu \mathrm{g}$ of protein was used to determine ASMase activity. Reaction was stopped after incubation at $37^{\circ} \mathrm{C}$ for $2 \mathrm{~h}$ and analysed using a fluorescence plate reader at $260 \mathrm{~nm}$ excitation and $460 \mathrm{~nm}$ emission.

Statistical analysis. Statistical analyses were conducted using Microsoft Office Excel 2007. Results were expressed as mean value \pm S.D. Differences between groups were evaluated using unpaired two-tailed Student's $t$-test.

\section{Conflict of Interest}

The authors declare no conflict of interest.
Acknowledgements. IP was a recipient of Northern Ireland HSC R\&D Office doctoral fellowship. IS was funded by British Lung Foundation. NC, FM and BW were funded by the Department for Education and Learning. AC, SB and NM were funded by Cancer Research UK. DF is a recipient of CR-UK Clinician Scientist Fellowship.

1. Jemal A, Murray T, Samuels A, Ghafoor A, Ward E, Thun MJ. Cancer statistics, 2003. CA Cancer J Clin 2003; 53: 5-26.

2. Fennell DA. Caspase regulation in non-small cell lung cancer and its potential for therapeutic exploitation. Clin Cancer Res 2005; 11: 2097-2105.

3. Klastersky J, Awada A. Milestones in the use of chemotherapy for the management of nonsmall cell lung cancer (NSCLC). Crit Rev Oncol Hematol 2012; 81: 49-57.

4. Belani CP, Langer C. First-line chemotherapy for NSCLC: an overview of relevant trials. Lung Cancer 2002; 38(Suppl 4): 13-19.

5. Schiller JH, Harrington D, Belani CP, Langer C, Sandler A, Krook J et al. Comparison of four chemotherapy regimens for advanced non-small-cell lung cancer. N Engl J Med 2002; 346: 92-98.

6. Paez JG, Janne PA, Lee JC, Tracy S, Greulich H, Gabriel S et al. EGFR mutations in lung cancer: correlation with clinical response to gefitinib therapy. Science 2004; 304: 1497-1500.

7. Lynch TJ, Bell DW, Sordella R, Gurubhagavatula S, Okimoto RA, Brannigan BW et al. Activating mutations in the epidermal growth factor receptor underlying responsiveness of non-small-cell lung cancer to gefitinib. N Engl J Med 2004; 350: 2129-2139.

8. Deng J, Shimamura T, Perera S, Carlson NE, Cai D, Shapiro Gl et al. Proapoptotic BH3only BCL-2 family protein BIM connects death signaling from epidermal growth factor receptor inhibition to the mitochondrion. Cancer Res 2007; 67: 11867-11875.

9. Costa DB, Halmos B, Kumar A, Schumer ST, Huberman MS, Boggon TJ et al. BIM mediates EGFR tyrosine kinase inhibitor-induced apoptosis in lung cancers with oncogenic EGFR mutations. PLoS Med 2007; 4: 1669-1679; discussion 1680.

10. Gong Y, Somwar R, Politi K, Balak M, Chmielecki J, Jiang X et al. Induction of BIM is essential for apoptosis triggered by EGFR kinase inhibitors in mutant EGFR-dependent lung adenocarcinomas. PLoS Med 2007; 4: e294.

11. Cragg MS, Kuroda J, Puthalakath $H$, Huang DC, Strasser A. Gefitinib-induced killing of NSCLC cell lines expressing mutant EGFR requires BIM and can be enhanced by BH3 mimetics. PLoS Med 2007; 4: 1681-1689; discussion 1690.

12. Soda M, Choi YL, Enomoto M, Takada S, Yamashita Y, Ishikawa S et al. Identification of the transforming EML4-ALK fusion gene in non-small-cell lung cancer. Nature 2007; 448: 561-566.

13. Brose MS, Volpe P, Feldman M, Kumar M, Rishi I, Gerrero R et al. BRAF and RAS mutations in human lung cancer and melanoma. Cancer Res 2002; 62: 6997-7000.

14. Cappuzzo F, Bemis L, Varella-Garcia M. HER2 mutation and response to trastuzumab therapy in non-small-cell lung cancer. N Engl J Med 2006; 354: 2619-2621.

15. Stephens P, Hunter C, Bignell G, Edkins S, Davies H, Teague J et al. Lung cancer: intragenic ERBB2 kinase mutations in tumours. Nature 2004; 431: 525-526.

16. Johnstone RW, Ruefli AA, Lowe SW. Apoptosis: a link between cancer genetics and chemotherapy. Cell 2002; 108: 153-164.

17. Hanahan D, Weinberg RA. Hallmarks of cancer: the next generation. Cell 4; 144: 646-674.

18. Youle RJ, Strasser A. The BCL-2 protein family: opposing activities that mediate cell death. Nat Rev Mol Cell Biol 2008; 9: 47.

19. Wei MC, Zong WX, Cheng EH, Lindsten T, Panoutsakopoulou V, Ross AJ et al. Proapoptotic BAX and BAK: a requisite gateway to mitochondrial dysfunction and death. Science 2001; 292: 727.

20. Apolinario RM, van der Valk P, de Jong JS, Deville W, van Ark-Otte J, Dingemans AM et al. Prognostic value of the expression of p53, bcl-2, and bax oncoproteins, and neovascularization in patients with radically resected non-small-cell lung cancer. J Clin Oncol 1997; 15: 2456.

21. Groeger AM, Esposito V, De Luca A, Cassandro R, Tonini G, Ambrogi V et al. Prognostic value of immunohistochemical expression of $\mathrm{p53}$, bax, $\mathrm{Bcl}-2$ and $\mathrm{Bcl}-\mathrm{xL}$ in resected nonsmall-cell lung cancers. Histopathology 2004; 44: 54.

22. Shabnam MS, Srinivasan R, Wali A, Majumdar S, Joshi K, Behera D. Expression of p53 protein and the apoptotic regulatory molecules Bcl-2, Bcl-XL, and Bax in locally advanced squamous cell carcinoma of the lung. Lung Cancer 2004; 45: 181.

23. Yaren A, Oztop I, Kargi A, Ulukus C, Onen A, Sanli A et al. Bax, bcl-2 and c-kit expression in non-small-cell lung cancer and their effects on prognosis. Int J Clin Pract 2006; 60: 675.

24. Berrieman HK, Smith L, O'Kane SL, Campbell A, Lind MJ, Cawkwell L. The expression of Bcl-2 family proteins differs between nonsmall cell lung carcinoma subtypes. Cancer 2005; 103: 1415.

25. Borner MM, Brousset P, Pfanner-Meyer B, Bacchi M, Vonlanthen S, Hotz MA et al. Expression of apoptosis regulatory proteins of the Bcl-2 family and p53 in primary resected non-small-cell lung cancer. Br J Cancer 1999; 79: 952.

26. Paul I, Savage KI, Blayney JK, Lamers E, Gately K, Kerr K et al. PARP inhibition induces BAX/BAK-independent synthetic lethality of BRCA1-deficient non-small cell lung cancer. $J$ Pathol 2011; 224: 564-574.

27. Ganten TM, Haas TL, Sykora J, Stahl H, Sprick MR, Fas SC et al. Enhanced caspase-8 recruitment to and activation at the DISC is critical for sensitisation of human hepatocellular 
carcinoma cells to TRAIL-induced apoptosis by chemotherapeutic drugs. Cell Death Differ 2004; 11(Suppl 1): S86-S96.

28. Lacour S, Micheau O, Hammann A, Drouineaud V, Tschopp J, Solary E et al. Chemotherapy enhances TNF-related apoptosis-inducing ligand DISC assembly in HT29 human colon cancer cells. Oncogene 2003; 22: 1807-1816.

29. Lacour S, Hammann A, Grazide S, Lagadic-Gossmann D, Athias A, Sergent 0 et al. Cisplatin-induced CD95 redistribution into membrane lipid rafts of HT29 human colon cancer cells. Cancer Res 2004; 64: 3593-3598.

30. Stegehuis JH, de Wilt LH, de Vries EG, Groen HJ, de Jong S, Kruyt FA. TRAIL receptor targeting therapies for non-small cell lung cancer: current status and perspectives. Drug Resist Updat 2010; 13: 2-15.

31. Rebillard A, Tekpli X, Meurette O, Sergent O, LeMoigne-Muller G, Vernhet L et al. Cisplatin-induced apoptosis involves membrane fluidification via inhibition of NHE1 in human colon cancer cells. Cancer Res 2007; 67: 7865-7874.

32. Crawford N, Chacko AD, Savage KI, McCoy F, Redmond K, Longley DB et al. Platinum resistant cancer cells conserve sensitivity to $\mathrm{BH} 3$ domains and obatoclax induced mitochondrial apoptosis. Apoptosis 2011; 16: 311-320.

33. Twentyman PR, Fox NE, Wright KA, Bleehen NM. Derivation and preliminary characterisation of adriamycin resistant lines of human lung cancer cells. $\mathrm{Br} J$ Cancer 1986; 53: 529-537.

34. Autret A, Martin SJ. Emerging role for members of the Bcl-2 family in mitochondria morphogenesis. Mol Cell 2009; 36: 355-363.
35. Wilson TR, Redmond KM, McLaughlin KM, Crawford N, Gately K, O'Byrne K et al. Procaspase 8 overexpression in non-small-cell lung cancer promotes apoptosis induced by FLIP silencing. Cell Death Differ 2009; 16: 1352-1361.

36. Gulbins E, Kolesnick R. Raft ceramide in molecular medicine. Oncogene 2003; 22: 7070-7077.

37. Grassme H, Cremesti A, Kolesnick R, Gulbins E. Ceramide-mediated clustering is required for CD95-DISC formation. Oncogene 2003; 22: 5457-5470.

38. Gulbins E. Regulation of death receptor signaling and apoptosis by ceramide. Pharmacol Res 2003; 47: 393-399.

39. Wilson TR, McLaughlin KM, McEwan M, Sakai H, Rogers KM, Redmond KM et al. c-FLIP: a key regulator of colorectal cancer cell death. Cancer Res 2007; 67: 5754-5762.

40. McCoy F, Hurwitz J, McTavish N, Paul I, Barnes C, O'Hagan B et al. Obatoclax induces Atg7-dependent autophagy independent of beclin-1 and BAX/BAK. Cell Death Dis 2010; 1 : e108

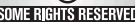

Cell Death and Disease is an open-access journal published by Nature Publishing Group. This work is licensed under the Creative Commons Attribution-NonCommercial-No Derivative Works 3.0 Unported License. To view a copy of this license, visit http://creativecommons.org/licenses/by-nc-nd/3.0/

Supplementary Information accompanies the paper on Cell Death and Disease website (http://www.nature.com/cddis) 